\title{
Remark on the vectorlike nature of electromagnetism and electric charge quantization
}

\author{
C. A de S. Pires \\ Instituto de Física Teórica, Universidade Estadual Paulista, Rua Pamplona 145, 01405-900 São Paulo, São Paulo, Brazil
}

(Received 25 June 1998; published 13 September 1999)

\begin{abstract}
In this work we study the structure of electromagnetic interactions and electric charge quantization in gauge theories of electroweak interactions based on semisimple groups. We show that in the standard model of electroweak interactions the structure of electromagnetic interactions is strongly correlated to the quantization pattern of electric charges. We examine these two questions also in all possible chiral bilepton gauge models of electroweak interactions. In all, we can explain the vectorlike nature of electromagnetic interactions and electric charge quantization together demanding nonvanishing fermion masses and anomaly cancellations. [S0556-2821(99)04917-6]

PACS number(s): 12.60.Cn, 12.90.+b
\end{abstract}

\section{INTRODUCTION}

Why nature arranges things so that electromagnetic interactions among fermions are vectorial and electric charge quantization (ECQ) comes with the pattern $Q_{\nu}=0, Q_{e}=$ $-e, Q_{u}=\frac{2}{3} e, Q_{d}=-\frac{1}{3} e$ is a question not altogether closed. $\mathrm{QED}$, the natural place to investigate these questions, is unable to explain them because of the arbitrariness of the $U(1)_{\text {em }}$ quantum numbers. Gauge theories of electroweak interactions based on a semisimple group face also the same difficulty as in QED: the U(1) factor compounding the semisimple groups.

In spite of this difficulty ECQ was analyzed in gauge theories of electroweak interactions. It was found that in some models the difficulties may be overcome by two types of constraints. One type comes from the requirement that some fermions be massive. In such models the fermions obtain masses through the Yukawa sector. Demanding this sector to be invariant under gauge symmetry we get constraints among the U(1) quantum numbers. These are the nonvanishing fermion mass constraints also called classical constraints. Another type of constraint comes from the requiriment of theoretical consistency of the model which means the model is free from anomalies. Anomaly cancellations [1] are expressed in terms of the relations among the U(1) quantum numbers. These are the quantum constraints. Using these two constraints ECQ can be explained in some extension of the standard model (SM) [2-8]. In this work we extend such an analysis to include the structure of the electromagnetic interactions. Such a study will be done in the SM with massless and massive neutrinos and in all possible chiral bilepton gauge (CBGM) versions.

This paper is organized as follows. In Sec. II we analyze the vectorlike nature of electromagnetism (VLNE) and ECQ in the SM with one and three generations. In Sec. III we extend our analysis to the case of CBGM. In Sec. IV we summarize our conclusions.

\section{ELECTRIC CHARGE QUANTIZATION AND THE VECTORLIKE NATURE OF ELECTROMAGNETISM IN THE FRAMEWORK OF THE STANDARD MODEL}

\section{A. The case of one generation}

In the SM with one generation the quarks and leptons come in the following representations:

$$
\begin{aligned}
& L_{L}=\left(\begin{array}{l}
\nu \\
e
\end{array}\right)_{L} \sim\left(1,2, Y_{L}\right), \quad e_{R} \sim\left(\mathbf{1}, 1, Y_{l}\right), \\
& Q_{L}=\left(\begin{array}{l}
u \\
d
\end{array}\right)_{L} \sim\left(\mathbf{3}, 2, Y_{q}\right), \quad u_{R} \sim\left(\mathbf{3}, 1, Y_{u}\right), \\
& d_{R} \sim\left(\mathbf{3}, 1, Y_{d}\right) .
\end{aligned}
$$

In order to break symmetry spontaneously and give masses to the gauge bosons $W^{ \pm}$and $Z^{0}$ we need to introduce a Higgs doublet $\phi \sim\left(\mathbf{1}, \mathbf{2}, Y_{\phi}\right)$ that acquires a vacuum expectation value

$$
\langle\phi\rangle_{0} \sim\left(\begin{array}{l}
0 \\
\mathbf{v}
\end{array}\right) .
$$

After the spontaneous symmetry breaking (SSB) and due to the mixing among $W^{3}$ and $B$ we find the following charge operator [4]:

$$
Q / a=T_{3}+b / a \frac{Y}{2},
$$

where $a=g / e \sin \theta_{W}$ and $b=g^{\prime} / e \cos \theta_{W}$. Since we want the generator $Q$ unbroken, $Q\langle\phi\rangle_{0}$ must be zero. With this condition we find $a=b Y_{\phi}$. Using the freedom in assigning the scale of the electric charge we set $a=1[2,4]$. Then we have $g \sin \theta_{W}=e$ and $g^{\prime} \cos \theta_{W}=e / Y_{\phi}$.

After these steps we find the following electromagnetic interactions among the fermions:

$$
\begin{aligned}
\mathcal{L}_{e m}= & -\frac{e}{4 Y_{\phi}}\left[2\left(Y_{L}+Y_{\phi}\right) \bar{\nu}_{L} \gamma^{\mu} \nu_{L}+\bar{e}\left(\left(-Y_{L}-Y_{l}+Y_{\phi}\right)\right.\right. \\
& \left.+\left(-Y_{L}+Y_{l}+Y_{\phi}\right) \gamma_{5}\right) \gamma^{\mu} e+\bar{u}\left(\left(-Y_{q}-Y_{u}-Y_{\phi}\right)\right. \\
& \left.+\left(-Y_{q}+Y_{u}-Y_{\phi}\right) \gamma_{5}\right) \gamma^{\mu} u+\bar{d}\left(\left(-Y_{q}-Y_{d}+Y_{\phi}\right)\right. \\
& \left.\left.+\left(-Y_{q}+Y_{d}+Y_{\phi}\right) \gamma_{5}\right) \gamma^{\mu} d\right] A_{\mu} .
\end{aligned}
$$

In order to give to fermions their masses we introduce the Yukawa interaction 


$$
-\mathcal{L}^{Y}=g^{l} \bar{L}_{L} \phi e_{R}+g^{u} \bar{Q}_{L} \widetilde{\phi} u_{R}+g^{d} \bar{Q}_{L} \phi d_{R}+\text { H.c. }
$$

By demanding $\mathrm{U}(1)_{Y}$ gauge invariance we find from Eq. (5)

$$
Y_{L}-Y_{l}-Y_{\phi}=0, \quad Y_{q}-Y_{u}+Y_{\phi}=0, \quad Y_{q}-Y_{d}-Y_{\phi}=0 .
$$

Substituting Eq. (6) in Eq. (4) we find the following structure to the electromagnetic interactions:

$$
\begin{aligned}
\mathcal{L}_{e m}= & -\frac{e}{2 Y_{\phi}}\left[\left(Y_{L}+Y_{\phi}\right) \bar{\nu}_{L} \gamma^{\mu} \nu_{L}+\bar{e}\left(-Y_{L}+Y_{\phi}\right) \gamma^{\mu} e\right. \\
& \left.-\left(Y_{q}+Y_{\phi}\right) \bar{u} \gamma^{\mu} u+\left(-Y_{q}+Y_{\phi}\right) \bar{d} \gamma^{\mu} d\right] A_{\mu} .
\end{aligned}
$$

Now we must impose the anomaly cancellation. The conditions in Eq. (6) leave only two nontrivial anomalies, which are sufficient to fix the hypercharges $Y_{L}$ and $Y_{q}$ :

$$
\begin{aligned}
{\left[\mathrm{SU}(2)_{L}\right]^{2} \mathrm{U}(1)_{Y} \Rightarrow Y_{q} } & =-\frac{1}{3} Y_{L}, \\
{\left[\mathrm{U}(1)_{Y}\right]_{Y}^{3} \Rightarrow Y_{L} } & =-Y_{\phi} \Rightarrow Y_{q}=\frac{1}{3} Y_{\phi} .
\end{aligned}
$$

Substituting Eq. (8) in Eq. (7) we obtain ECQ and the VLNE

$$
\mathcal{L}_{e m}=e \bar{e} \gamma^{\mu} e A_{\mu}-\frac{2 e}{3} \bar{u} \gamma^{\mu} u A_{\mu}+\frac{e}{3} \bar{d} \gamma^{\mu} d A_{\mu}
$$

Cancellation of anomalies and demanding the fermions are massive one obtains ECQ and the VLNE in the SM with one generation and massless neutrinos.

Adding a right-handed Dirac neutrino, $\nu_{R} \sim\left(\mathbf{1}, 2, Y_{\nu}\right)$, to the SM we get the following electromagnetic interaction:

$$
-\frac{e}{4 Y_{\phi}} \bar{\nu}_{l}\left(\left(Y_{L}+Y_{\nu}+Y_{\phi}\right)+\left(Y_{L}-Y_{\nu}+Y_{\phi}\right) \gamma_{5}\right) \gamma^{\mu} \nu_{l} A_{\mu}
$$

Its Yukawa term is $\bar{L}_{L} \widetilde{\phi} \nu_{R}$. Its $\mathrm{U}(1)_{Y}$ gauge invariance provides the following relation: $-Y_{L}+Y_{\nu}-Y_{\phi}=0$, which cancels the axial term in Eq. (10). But now only one nontrivial anomaly constraint remains: $\left[\mathrm{SU}(2)_{L}\right]^{2} \mathrm{U}(1)_{Y}$. Then we have two arbitrary hypercharges from the gauge invariance of the Yukawa sector and one constraint from the anomaly cancellation. In this case we have no ECQ. This result is the dequantization effect [2-7]. Nevertheless we have the VLNE automatically

$$
\begin{aligned}
\mathcal{L}_{e m}= & -\frac{e}{2 Y_{\phi}}\left[\left(Y_{L}+Y_{\phi}\right) \bar{\nu} \gamma^{\mu} \nu+\left(-Y_{L}+Y_{\phi}\right) \bar{e} \gamma^{\mu} e\right. \\
& \left.+\left(\frac{Y_{L}}{3}+Y_{\phi}\right) \bar{u} \gamma^{\mu} u+\left(-\frac{Y_{L}}{3}+Y_{\phi}\right) \bar{d} \gamma^{\mu} d\right] A_{\mu} .
\end{aligned}
$$

Babu and Mohapatra made the important observation in Ref. [5] that if we suppose that the neutrino is a Majorana particle we can fix all the hypercharges restoring thus ECQ and consequently the VLNE.

\section{B. The case of three generations}

With three generations the representation content is

$$
\begin{aligned}
& L_{a_{L}}=\left(\begin{array}{c}
\nu_{a} \\
e_{a}
\end{array}\right)_{L} \sim\left(\mathbf{1}, 2, Y_{L_{a}}\right), \quad e_{a_{R}} \sim\left(\mathbf{1}, \mathbf{1}, Y_{l_{a}}\right), \\
& Q_{a_{L}}=\left(\begin{array}{c}
u_{a} \\
d_{a}
\end{array}\right)_{L} \sim\left(\mathbf{3}, 2, Y_{q_{a}}\right), \quad u_{a_{R}} \sim\left(\mathbf{3}, \mathbf{1}, Y_{u_{a}}\right), \\
& d_{a_{R}} \sim\left(\mathbf{3}, \mathbf{1}, Y_{d_{a}}\right) .
\end{aligned}
$$

With $a=1,2,3$ being the flavor index. Now we have the following structure for the electromagnetic interactions:

$$
\begin{aligned}
\mathcal{L}_{e m}= & -\frac{e}{4 Y_{\phi}} \sum_{a}^{3}\left[2\left(Y_{L_{a}}+Y_{\phi}\right) \bar{\nu}_{a_{L}} \gamma^{\mu} \nu_{i_{L}}\right. \\
& +\bar{e}_{a}\left(\left(-Y_{L_{a}}-Y_{l_{a}}+Y_{\phi}\right)+\left(-Y_{L_{a}}+Y_{l_{a}}+Y_{\phi}\right) \gamma_{5}\right) \gamma^{\mu} e_{a} \\
& +\bar{u}_{a}\left(\left(-Y_{q_{a}}-Y_{u_{a}}-Y_{\phi}\right)+\left(-Y_{q_{a}}+Y_{u_{a}}-Y_{\phi}\right) \gamma_{5}\right) \gamma^{\mu} u_{a} \\
& +\bar{d}_{a}\left(\left(-Y_{q_{a}}-Y_{d_{a}}+Y_{\phi}\right)\right. \\
& \left.\left.+\left(-Y_{q_{a}}+Y_{d_{a}}+Y_{\phi}\right) \gamma_{5}\right) \gamma^{\mu} d_{a}\right] A_{\mu} .
\end{aligned}
$$

The Yukawa sector takes the following form [4]:

$$
\begin{aligned}
-\mathcal{L}^{Y}= & \sum_{a, b}^{1,2,3}\left[g_{a a}^{l} \bar{L}_{a_{L}} \phi e_{a_{R}}+g_{a b}^{d} \bar{Q}_{a_{L}} \phi d_{b_{R}}+g_{a b}^{u} \bar{Q}_{a_{L}} \widetilde{\phi} u_{b_{R}}\right] \\
& + \text { H.c. }
\end{aligned}
$$

From its $\mathrm{U}(1)_{Y}$ gauge invariance we find the following relations among the hypercharges:

$$
\begin{aligned}
& Y_{L_{a}}-Y_{l_{a}}-Y_{\phi}=0, \quad Y_{q_{a}}-Y_{u_{b}}-Y_{\phi}=0, \\
& Y_{q_{a}}-Y_{d_{b}}+Y_{\phi}=0 .
\end{aligned}
$$

From the two last terms above we obtain

$$
Y_{q_{a}}=Y_{q}, \quad Y_{u_{a}}=Y_{u}, \quad Y_{d_{a}}=Y_{d},
$$

which leads Eq. (15) to

$$
Y_{L_{a}}-Y_{l_{a}}-Y_{\phi}=0, \quad Y_{q}-Y_{u}-Y_{\phi}=0, \quad Y_{q}-Y_{d}+Y_{\phi}=0 .
$$

Substituting Eq. (17) into Eq. (13) we obtain the following electromagnetic interaction among fermions:

$$
\begin{aligned}
\mathcal{L}_{e m}= & -\frac{e}{2 Y_{\phi}} \sum_{a}^{3}\left[\left(Y_{L_{a}}+Y_{\phi}\right) \bar{\nu}_{a_{L}} \gamma^{\mu} \nu_{a_{L}} A_{\mu}\right. \\
& +\bar{e}_{a}\left(-Y_{L_{a}}+Y_{\phi}\right) \gamma^{\mu} e_{a} A_{\mu}-\bar{u}_{a}\left(Y_{q}+Y_{\phi}\right) \gamma^{\mu} u_{a} A_{\mu} \\
& \left.+\bar{d}_{a}\left(-Y_{q}+Y_{\phi}\right) \gamma^{\mu} d_{a} A_{\mu}\right] .
\end{aligned}
$$

After this we have only two nontrivial anomaly constraints 


$$
\begin{aligned}
{\left[\mathrm{SU}(2)_{L}\right]^{2} \mathrm{U}(1)_{Y} \Rightarrow } & 9 Y_{q}+\sum_{a}^{3} Y_{L_{a}}=0, \\
{\left[\mathrm{U}(1)_{Y}\right]_{Y}^{3} \Rightarrow } & 18 Y_{q}^{3}-9 Y_{u}^{3}-9 Y_{d}^{3} \\
& +\sum_{a}^{3}\left(2 Y_{L_{a}}^{3}-Y_{l_{a}}^{3}\right)=0 .
\end{aligned}
$$

These two constraints are insufficient for fixing the four arbitrary hypercharges in Eq. (18). Different from the SM with one generation, we have neither an explanation to ECQ nor to the VLNE. This is the dequantization effect [2-7]. Nevertheless we have through Eq. (18) a correlation among the quantization pattern and the structure of the electromagnetic interactions. Such correlation permits us to conclude that nature arranges things so that the electric charge quantization comes with the quantization pattern $Q_{\nu}=0, Q_{e}=-e, Q_{u}$ $=\frac{2}{3} e, Q_{d}=-\frac{1}{3} e$ because the nature of electromagnetic interactions is vectorial. Thus if we wish to explain ECQ we need to take as constraints the nonvanishing fermion masses, anomaly cancellations and the VLNE.

Let us suppose a Dirac-like massive neutrino. In this case also we have the dequantization effect [2-4]. Nevertheless the VLNE is automatic

$$
\begin{aligned}
\mathcal{L}_{e m}= & -\frac{e}{2 Y_{\phi}} \sum_{a}^{3}\left[\left(Y_{L}+Y_{\phi}\right) \bar{\nu}_{a} \gamma^{\mu} \nu_{a} A_{\mu}\right. \\
& +\bar{e}_{a}\left(-Y_{L}+Y_{\phi}\right) \gamma^{\mu} e_{a} A_{\mu}-\bar{u}_{a}\left(-\frac{Y_{L}}{3}+Y_{\phi}\right) \gamma^{\mu} u_{a} A_{\mu} \\
& \left.+\bar{d}_{a}\left(-\frac{Y_{L}}{3}+Y_{\phi}\right) \gamma^{\mu} d_{a} A_{\mu}\right]
\end{aligned}
$$

where $Y_{L_{1}}=Y_{L_{2}}=Y_{L_{3}}=Y_{L}[4]$.

If we suppose a Majorana-like massive neutrino we must restore ECQ and the VLNE as in the case of the SM with one generation $[2,3]$.

\section{ELECTRIC CHARGE QUANTIZATION AND THE VECTORLIKE NATURE OF ELECTROMAGNETISM IN CHIRAL BILEPTON GAUGE MODELS}

Chiral bilepton gauge models are extensions of the $\mathrm{SU}(3)_{C} \otimes \mathrm{SU}(2)_{L} \otimes \mathrm{U}(1)_{Y}$ symmetry group to the $G_{3 X 1}$ $=\mathrm{SU}(3)_{C} \otimes \mathrm{SU}(X)_{L} \otimes \mathrm{U}(1)_{N}$ one, with $X=3,4 . X=3$ gives us the simplest versions and $X=4$ the largest version. Whether or not we consider exotic leptons we can have only two independent simplest versions of CBGM $[9,10]$ and one largest version [11]. Their key features are bilepton gauge bosons with lepton number $L= \pm 2$ and exotic quarks. They give in some sense an answer to the family problem [12] because they require a minimum of three families to cancel anomalies. They are multi-Higgs models, nevertheless FCNC with the standard neutral gauge boson strongly sup- pressed [13]. Some studies of their phenomenology were done in [14].

\section{A. Version A}

Here we analyze the VLNE and ECQ in a CBGM based in the $\mathrm{SU}(3)_{C} \otimes \mathrm{SU}(3)_{L} \otimes \mathrm{U}(1)_{N}$ symmetry which has as electric charge operator the following linear combinations of their diagonal generators [9]:

$$
Q=\frac{1}{2}\left(\lambda_{3}-\sqrt{3} \lambda_{8}\right)+b N,
$$

where $N$ is the generator operator of the $\mathrm{U}(1)_{N}$ group and $\lambda_{3}$ and $\lambda_{8}$ are the two diagonal Gell-mann matrices.

The mininal set of scalars necessary to give correct masses to the fermions and bosons are three triplets and one sextet. They get a vacuum expectation value different from zero and transform by $\mathrm{SU}(3)_{C} \otimes \mathrm{SU}(3)_{L} \otimes \mathrm{U}(1)_{N}$ in the following manner:

$$
\begin{aligned}
& \langle\eta\rangle_{0}=\left(\begin{array}{c}
\mathrm{v}_{\eta} \\
0 \\
0
\end{array}\right) \sim\left(\mathbf{1}, \mathbf{3}, N_{\eta}\right), \quad\langle\rho\rangle_{0}=\left(\begin{array}{c}
0 \\
\mathrm{v}_{\rho} \\
0
\end{array}\right) \sim\left(\mathbf{1}, \mathbf{3}, N_{\rho}\right) \\
& \langle\chi\rangle_{0}=\left(\begin{array}{c}
0 \\
0 \\
\mathrm{v}_{\chi}
\end{array}\right) \sim\left(\mathbf{1}, \mathbf{3}, N_{\chi}\right) \\
& \langle S\rangle_{0}=\left(\begin{array}{ccc}
0 & 0 & 0 \\
0 & 0 & \mathrm{v}_{\sigma_{2}} \\
0 & \mathrm{v}_{\sigma_{2}} & 0
\end{array}\right) \sim\left(\mathbf{1}, \mathbf{3}, N_{S}\right)
\end{aligned}
$$

Requiring the electric charge operator annihilates the vacuum of the scalars we set

$$
N_{\eta}=0, \quad b=\frac{1}{N_{\rho}}, \quad N_{\chi}=-N_{\rho}, \quad N_{S}=0 .
$$

After these steps the electric charge operator acquires the following form:

$$
Q=\frac{1}{2}\left(\lambda_{3}-\sqrt{3} \lambda_{8}\right)+\frac{N}{N_{\rho}} .
$$

The fermions come in the following representations:

$$
\begin{aligned}
& L_{a_{L}}=\left(\begin{array}{c}
\nu_{a} \\
e_{a} \\
e_{a}^{c}
\end{array}\right)_{L} \sim\left(\mathbf{1}, \mathbf{3}, N_{L_{a}}\right), \\
& Q_{1_{L}}=\left(\begin{array}{c}
u_{1} \\
d_{1} \\
J_{1}
\end{array}\right)_{L} \sim\left(\mathbf{3}, \mathbf{3}, N_{Q_{1}}\right), \\
& u_{1 R} \sim\left(\mathbf{3}, \mathbf{1}, N_{u_{1}}\right), \quad d_{1_{R}} \sim\left(\mathbf{3}, \mathbf{1}, N_{d_{1}}\right),
\end{aligned}
$$




$$
\begin{aligned}
& J_{R_{1}} \sim\left(\mathbf{3}, \mathbf{1}, N_{J_{1}}\right), \\
& Q_{i_{L}}=\left(\begin{array}{c}
d_{i} \\
-u_{i} \\
J_{i}
\end{array}\right)_{L} \sim\left(\mathbf{3}, \mathbf{3}^{*}, N_{Q_{i}}\right), \\
& d_{i_{R}} \sim\left(\mathbf{3}, \mathbf{1}, N_{d_{i}}\right), \quad u_{i_{R}} \sim\left(\mathbf{3}, \mathbf{1}, N_{u_{i}}\right),
\end{aligned}
$$

$$
J_{i_{R}} \sim\left(3,1, N_{J_{i}}\right),
$$

with $a=1,2,3$ and $i=2,3$ being flavor indices. There are no lepton singlets. The quarks $u$ 's and $d$ 's are the usual ones with $J$ 's being the exotic quarks.

After $\quad \mathrm{SSB} \quad \mathrm{SU}(3)_{C} \otimes \mathrm{SU}(3)_{L} \otimes \mathrm{U}(1)_{N} \rightarrow \mathrm{SU}(3)_{C}$ $\otimes \mathrm{U}(1)_{e m}$ we find the following structure for the electromagnetic interactions:

$$
\begin{aligned}
\mathcal{L}_{e m}= & -\frac{e}{2 N_{\rho}} \sum_{a}^{1,2,3} \sum_{i}^{2,3}\left[2 N_{L_{a}} \bar{\nu}_{a_{L}} \gamma^{\mu} \nu_{a_{L}}+2 \bar{e}_{a}\left(-N_{\rho}+N_{L_{a}} \gamma_{5}\right) \gamma^{\mu} e_{a}\right. \\
& +\bar{u}_{1}\left(\left(N_{Q_{1}}+N_{u_{1}}\right)-\left(-N_{Q_{1}}+N_{u_{1}}\right) \gamma_{5}\right) \gamma^{\mu} u_{1}+\bar{u}_{i}\left(\left(N_{Q_{i}}+N_{u_{i}}+N_{\rho}\right)-\left(N_{Q_{i}}-N_{u_{i}}+N_{\rho}\right) \gamma_{5}\right) \gamma^{\mu} u_{i} \\
& +\bar{d}_{1}\left(\left(N_{Q_{1}}+N_{d_{1}}-N_{\rho}\right)-\left(N_{Q_{1}}-N_{d_{1}}-N_{\rho}\right) \gamma_{5}\right) \gamma^{\mu} d_{1}+\bar{d}_{i}\left(\left(N_{Q_{i}}+N_{d_{i}}\right)-\left(N_{Q_{i}}-N_{d_{i}}\right) \gamma_{5}\right) \gamma^{\mu} d_{i} \\
& \left.+\bar{J}_{1}\left(\left(N_{Q_{1}}+N_{J_{1}}+N_{\rho}\right)-\left(N_{Q_{1}}+N_{J_{1}}-N_{\rho}\right) \gamma_{5}\right) \gamma^{\mu} J_{1}+\bar{J}_{i}\left(\left(N_{Q_{i}}+N_{J_{i}}-N_{\rho}\right)-\left(N_{Q_{i}}-N_{J_{i}}-N_{\rho}\right) \gamma_{5}\right) \gamma^{\mu} J_{i}\right] A_{\mu} .
\end{aligned}
$$

From the $\mathrm{U}(1)_{N}$ invariance of the Yukawa sector [15]

$$
\begin{aligned}
-\mathcal{L}_{Y}= & \frac{1}{2} G_{a b} \bar{L}_{a L}^{c} S^{*} L_{b L}+\lambda_{1} \bar{Q}_{1 L} J_{1 R} \chi+\lambda_{i j} \bar{Q}_{i L} J_{j R} \chi^{*} \\
& +\lambda_{1 a}^{\prime} \bar{Q}_{1 L} d_{a R} \rho+\lambda_{i a}^{\prime} \bar{Q}_{i L} u_{a R} \rho^{*}+\lambda_{1 a}^{\prime \prime} \bar{Q}_{1 L} u_{a R} \eta \\
& +\lambda_{i a}^{\prime \prime} \bar{Q}_{i L} d_{a R} \eta^{*}+\text { H.c. },
\end{aligned}
$$

we find the following relations between the $N$ quantum numbers [8]:

$$
\begin{aligned}
& N_{u_{1}}=N_{u_{2}}=N_{u_{3}}=N_{u}, \\
& N_{d_{1}}=N_{d_{2}}=N_{d_{3}}=N_{d}, \\
& N_{Q_{2}}=N_{Q_{3}}=N_{Q}, \\
& N_{J_{2}}=N_{J_{3}}=N_{J},
\end{aligned}
$$

and

$$
\begin{aligned}
& N_{L_{1}}=N_{L_{2}}=N_{L_{3}}=0, \\
& N_{J}=N_{Q}-N_{\rho}, \\
& N_{d}=N_{Q}, \\
& N_{u}=N_{Q}+N_{\rho}, \\
& N_{J_{1}}=N_{Q}+2 N_{\rho}, \\
& N_{Q_{1}}=N_{Q}+N_{\rho} .
\end{aligned}
$$

Substituting these relations in Eq. (26) we obtain the following electromagnetic interactions:

$$
\begin{aligned}
\mathcal{L}_{e m}= & e \bar{e}_{a} \gamma^{\mu} e_{a} A_{\mu}-\frac{e}{N_{\rho}}\left[\left(N_{Q}+N_{\rho}\right) \bar{u}_{a} \gamma^{\mu} u_{a}+N_{Q} \bar{d}_{a} \gamma^{\mu} d_{a}\right. \\
& \left.+\left(N_{Q}+2 N_{\rho}\right) \bar{J}_{1} \gamma^{\mu} J_{1}+\left(N_{Q}-N_{\rho}\right) \bar{J}_{i} \gamma^{\mu} J_{i}\right] A_{\mu} .
\end{aligned}
$$

Note that the classical constraints alone provide the VLNE and ECQ among the leptons.

After the relations in Eq. (29) only one nontrivial anomaly constraint remains

$$
\begin{aligned}
& {\left[\mathrm{SU}(3)_{L}\right]^{2} \mathrm{U}(1)_{N} \Rightarrow 3 N_{Q_{1}}+3 N_{Q_{2}}+3 N_{Q_{3}}+N_{L_{1}}+N_{L_{2}}+N_{L_{3}}} \\
& \quad=0
\end{aligned}
$$

which together with the relations in Eq. (29) gives $N_{Q}=$ $-N_{\rho} / 3$. This fixes uniquely the $N$ 's quantum numbers as a function of $N_{\rho}$, furnishing thereby electric charge quantization of all fermions and the VLNE

$$
\begin{aligned}
\mathcal{L}_{e m}= & e \bar{e}_{a} \gamma^{\mu} e_{a} A_{\mu}-\frac{2 e}{3} \bar{u}_{a} \gamma^{\mu} u_{a} A_{\mu}+\frac{e}{3} \bar{d}_{a} \gamma^{\mu} d_{a} A_{\mu} \\
& -\frac{5 e}{3} \bar{J}_{1} \gamma^{\mu} J_{1} A_{\mu}+\frac{4 e}{3} \bar{J}_{i} \gamma^{\mu} J_{i} A_{\mu} .
\end{aligned}
$$

Let us now analyze this model with massive neutrinos. In the case of a Majorana neutrino, it is sufficient to modify the sextet of scalars to

$$
\langle S\rangle_{0}=\left(\begin{array}{lll}
\mathrm{v}_{\sigma_{1}} & 0 & 0 \\
0 & 0 & \mathrm{v}_{\sigma_{2}} \\
0 & \mathrm{v}_{\sigma_{2}} & 0
\end{array}\right) \sim\left(\mathbf{1}, \mathbf{3}, N_{S}\right) .
$$

This does not change the above steps. So the result in Eq. (32) remains the same with or without Majorana neutrinos. 
If we add right-handed neutrinos, $\nu_{a_{R}} \sim\left(\mathbf{1}, \mathbf{1}, N_{R_{a}}\right)$, their electromagnetic interaction become

$$
\mathcal{L}_{e m}^{\nu}=-\frac{e}{N_{\rho}} \sum_{a}^{3}\left(N_{L_{a}}+N_{R_{a}}\right) \bar{\nu}_{a} \gamma^{\mu} \nu_{a} A^{\mu} .
$$

From their Yukawa interactions $\bar{L}_{a_{L}} \eta \nu_{a_{R}}$, we find $N_{R_{a}}$ $-N_{L_{a}}=0$, which together with Eq. (29) gives $N_{R_{a}}=0$, annulling their electromagnetic interactions. In short, in this simplest CBGM version, we explain, with or without massives neutrinos, ECQ and the VLNE.

\section{B. Version B}

This is another possible variant of the simplest CBGM versions. Its Higgs sector is more economic than the one in the first version. It presents Dirac-like massive neutrinos inevitably in tree level. Its fermion content is the following:

$$
\begin{aligned}
& L_{a_{L}}=\left(\begin{array}{c}
\nu_{a} \\
e_{a} \\
\nu_{a}^{c}
\end{array}\right)_{L} \sim\left(\mathbf{1}, \mathbf{3}, N_{L_{a}}\right), \quad e_{a_{R}} \sim\left(\mathbf{1}, \mathbf{1}, N_{R_{a}}\right), \\
& Q_{\alpha_{L}}=\left(\begin{array}{c}
d_{\alpha} \\
-u_{\alpha} \\
J_{\alpha}
\end{array}\right)_{L} \sim\left(\mathbf{3}, \mathbf{3}^{*}, N_{Q_{\alpha}}\right), \\
& u_{\alpha_{R}} \sim\left(\mathbf{3}, \mathbf{1}, N_{u_{\alpha}}\right), \quad d_{\alpha_{R}} \sim\left(\mathbf{3}, \mathbf{1}, N_{d_{\alpha}}\right), \\
& J_{R_{\alpha}} \sim\left(\mathbf{3}, \mathbf{1}, N_{J_{\alpha}}\right), \\
& Q_{3 L}=\left(\begin{array}{c}
u_{3} \\
d_{3} \\
J_{3}
\end{array}{ }_{L} \sim\left(\mathbf{3}, \mathbf{3}, N_{Q_{3}}\right),\right.
\end{aligned}
$$

$$
\begin{aligned}
& d_{3_{R}} \sim\left(\mathbf{3}, \mathbf{1}, N_{d_{3}}\right), \quad u_{3_{R}} \sim\left(\mathbf{3}, \mathbf{1}, N_{u_{3}}\right), \\
& J_{3 R} \sim\left(\mathbf{3}, \mathbf{1}, N_{J_{3}}\right),
\end{aligned}
$$

with $a=1,2,3$ and $\alpha=1,2$ being the flavor indices.

Its electric charge operator takes the following linear combination [10]:

$$
Q=\frac{1}{2}\left(\lambda_{3}-\frac{1}{\sqrt{3}} \lambda_{8}\right)+b^{\prime} N .
$$

We need three triplets of scalars to break spontaneously the symmetry and give mass to the fermions. Their vacuum expectation value is different from zero and transforms by $\mathrm{SU}(3)_{C} \otimes \mathrm{SU}(3)_{L} \otimes \mathrm{U}(1)_{N}$ in the following manner:

$$
\begin{aligned}
& \langle\eta\rangle_{0}=\left(\begin{array}{c}
\mathrm{v}_{\eta} \\
0 \\
0
\end{array}\right) \sim\left(\mathbf{1}, \mathbf{3}, N_{\eta}\right), \quad\langle\rho\rangle_{0}=\left(\begin{array}{c}
0 \\
\mathrm{v}_{\rho} \\
0
\end{array}\right) \sim\left(\mathbf{1}, \mathbf{3}, N_{\rho}\right), \\
& \langle\chi\rangle_{0}=\left(\begin{array}{c}
0 \\
0 \\
\mathbf{v}_{\chi}
\end{array}\right) \sim\left(\mathbf{1}, \mathbf{3}, N_{\chi}\right) .
\end{aligned}
$$

With the requirement that the electric charge operator annihilates the vacuum of the scalars we set

$$
N_{\rho}=-2 N_{\eta}, \quad N_{\chi}=N_{\eta}, \quad b^{\prime}=-\frac{1}{3 N_{\eta}} .
$$

After the break of the symmetry $\mathrm{SU}(3)_{C} \otimes \mathrm{SU}(3)_{L}$ $\otimes \mathrm{U}(1)_{N} \rightarrow \mathrm{SU}(3)_{C} \otimes \mathrm{U}(1)_{e m}$ we find the following structure for their electromagnetic interactions:

$$
\begin{aligned}
\mathcal{L}_{e m}= & -\frac{e}{6 N_{\eta}} \sum_{a}^{1,2,3} \sum_{\alpha}^{1,2}\left[\left(N_{L_{a}}-N_{\eta}\right) \bar{\nu}_{a} \gamma_{5} \gamma^{\mu} \nu_{a}+\bar{e}_{a}\left(\left(-N_{L_{a}}-N_{R_{a}}-2 N_{\eta}\right)-\left(-N_{L_{a}}+N_{R_{a}}-2 N_{\eta}\right) \gamma_{5}\right) \gamma^{\mu} e_{a}\right. \\
& +\bar{u}_{\alpha}\left(\left(-N_{Q_{\alpha}}-N_{u_{\alpha}}+2 N_{\eta}\right)-\left(-N_{Q_{\alpha}}+N_{u_{\alpha}}+2 N_{\eta}\right) \gamma_{5}\right) \gamma^{\mu} u_{\alpha}+\bar{u}_{3}\left(\left(-N_{Q_{3}}-N_{u_{3}}+N_{\eta}\right)\right. \\
& \left.-\left(-N_{Q_{3}}+N_{u_{3}}+N_{\eta}\right) \gamma_{5}\right) \gamma^{\mu} u_{3}+\bar{d}_{\alpha}\left(\left(-N_{Q_{\alpha}}-N_{d_{\alpha}}-N_{\eta}\right)-\left(-N_{Q_{\alpha}}+N_{d_{\alpha}}-N_{\eta}\right) \gamma_{5}\right) \gamma^{\mu} d_{\alpha} \\
& +\bar{d}_{3}\left(\left(-N_{Q_{3}}-N_{d_{3}}-2 N_{\eta}\right)-\left(-N_{Q_{3}}+N_{d_{3}}-2 N_{\eta}\right) \gamma_{5}\right) \gamma^{\mu} d_{3}+\bar{J}_{\alpha}\left(\left(-N_{Q_{\alpha}}-N_{J_{\alpha}}-N_{\eta}\right)\right. \\
& \left.\left.-\left(-N_{Q_{\alpha}}+N_{J_{\alpha}}-N_{\eta}\right) \gamma_{5}\right) \gamma^{\mu} J_{\alpha}+\bar{J}_{3}\left(\left(-N_{Q_{3}}-N_{J_{3}}+N_{\eta}\right)-\left(-N_{Q_{3}}+N_{J_{3}}+N_{\eta}\right) \gamma_{5}\right) \gamma^{\mu} J_{3}\right] A_{\mu} .
\end{aligned}
$$

The Yukawa sector here is [10]

$$
\begin{aligned}
-\mathcal{L}_{Y}= & G_{a b} \epsilon^{l m n}\left(\bar{L}_{a_{L}}\right)_{l}\left(L_{b_{L}}\right)_{m}\left(\rho^{*}\right)_{n}+G_{a b}^{\prime} \bar{L}_{a_{L}} e_{b_{R}} \rho+\lambda_{1} \bar{Q}_{3_{L}} J_{3_{R}} \chi+\lambda_{2 \alpha \beta} \bar{Q}_{\alpha_{L}} J_{\beta_{R}} \chi^{*}+\lambda_{1 a} \bar{Q}_{3_{L}} d_{a_{R}} \rho \\
& +\lambda_{2 \alpha a} \bar{Q}_{\alpha L} u_{a_{R}} \rho^{*}+\lambda_{3 a} \bar{Q}_{3_{L}} u_{a_{R}} \eta+\lambda_{4 \alpha a} \bar{Q}_{\alpha_{L}} d_{a_{R}} \eta^{*}+\text { H.c. },
\end{aligned}
$$


with $a, b=1,2,3$ and $\alpha, \beta=1,2$. From $\mathrm{U}(1)_{N}$ invariance, this sector supplies us with the following relations between the $N$ quantum numbers:

$$
\begin{aligned}
& N_{u_{1}}=N_{u_{2}}=N_{u_{3}}=N_{u}, \\
& N_{d_{1}}=N_{d_{2}}=N_{d_{3}}=N_{d}, \\
& N_{Q_{1}}=N_{Q_{2}}=N_{Q}, \\
& N_{J_{1}}=N_{J_{2}}=N_{J},
\end{aligned}
$$

and

$$
\begin{aligned}
& N_{L_{1}}=N_{L_{2}}=N_{L_{3}}=N_{\eta}, \\
& N_{R_{1}}=N_{R_{2}}=N_{R_{3}}=3 N_{\eta}, \\
& N_{u}=N_{Q}-2 N_{\eta}, \\
& N_{d}=N_{Q}+N_{\eta}, \\
& N_{Q_{3}}=N_{Q}-N_{\eta}, \\
& N_{J_{3}}=N_{Q}-2 N_{\eta}, \\
& N_{J}=N_{Q}+N_{\eta} .
\end{aligned}
$$

Substituting these relations into Eq. (39) we obtain

$$
\begin{aligned}
\mathcal{L}_{e m}= & e \bar{e}_{a} \gamma^{\mu} e_{a} A_{\mu}-\frac{e}{3 N_{\eta}}\left[\left(-N_{Q}+2 N_{\eta}\right) \bar{u}_{a} \gamma^{\mu} u_{a}\right. \\
& -\left(N_{Q}+N_{\eta}\right) \bar{d}_{a} \gamma^{\mu} d_{a}+\left(N_{Q}+2 N_{\eta}\right) \bar{J}_{3} \gamma^{\mu} J_{3} \\
& \left.-\left(N_{Q}+N_{\eta}\right) \bar{J}_{\alpha} \gamma^{\mu} J_{\alpha}\right] A_{\mu} .
\end{aligned}
$$

Note that, as in version A, the classical constraints lead to ECQ and the VLNE in the leptonic sector while in the quark sector they lead only to the VLNE.

Again, as in the previous section, only one nontrivial anomaly constraint remains

$$
\begin{aligned}
& {\left[\mathrm{SU}(3)_{L}\right]^{2} \mathrm{U}(1)_{N} \Rightarrow 3 N_{Q_{1}}+3 N_{Q_{2}}+3 N_{Q_{3}}+N_{L_{1}}+N_{L_{2}}+N_{L_{3}}} \\
& \quad=0,
\end{aligned}
$$

which together with the relations in Eq. (42) gives $N_{Q}=0$. This result fixes uniquely the $N$ 's quantum numbers in function $N_{\eta}$ explaining ECQ for all fermions and leading to the VLNE

$$
\begin{aligned}
\mathcal{L}_{e m}= & e \bar{e}_{a} \gamma^{\mu} e_{a} A_{\mu}-\frac{2 e}{3} \bar{u}_{a} \gamma^{\mu} u_{a} A_{\mu}+\frac{e}{3} \bar{d}_{a} \gamma^{\mu} d_{a} A_{\mu} \\
& +\frac{e}{3} \bar{J}_{\alpha} \gamma^{\mu} J_{\alpha} A_{\mu}-\frac{2 e}{3} \bar{J}_{3} \gamma^{\mu} J_{3} A_{\mu} .
\end{aligned}
$$

The largest $\mathrm{CBGM}$ version is based on the $\mathrm{SU}(3)_{C}$ $\otimes \mathrm{SU}(4)_{L} \otimes \mathrm{U}(1)_{N}$ symmetry group [11]. In it ECQ takes place in the same way as in the first simplest version, as was shown recently in [16]. Thus the VLNE and ECQ must be explained in the same way as in the simplest version A. We close this section by saying that in CBGM inevitably we explain ECQ and the structure of the electromagnetic interactions.

\section{CONCLUSIONS}

In this paper we have examined the correlation among ECQ and VLNE in some gauge theory of electroweak interactions in order to understand the structure of one of the four fundamental forces of nature. Depending on the model and on their representation content ECQ and the VLNE are strongly correlated. This is the case with the SM with three generations and massless neutrinos. In this case, through the classical and quantum constraints, we do not explain neither the ECQ nor the VLNE. Nevertheless we can understand why the electric charge is quantized with the pattern required by nature through a correlation among ECQ and the VLNE obtained in Eq. (18). There we can see that such a required pattern occurs because QED is vectorial. Also such a correlation shows that if we wish to explain ECQ we must require as constraints the nonvanishing fermion masses, the anomaly cancellations and the VLNE. In the case of Dirac-like massive neutrinos we lose such correlations in the sense that we have the VLNE but not ECQ. In the case of Majorana-like massive neutrinos we restore the VLNE and ECQ. In the chiral bilepton gauge model we can explain ECQ and the VLNE together. This takes place in all versions, with or without massive neutrinos, through the nonvanishing fermion masses and anomaly cancellations. These results make CBGM an interesting extension of the SM. Principally we hope that a final theory of matter and forces explains the VLNE and ECQ together.

\section{ACKNOWLEDGMENTS}

I thank J. C. Montero, V. Pleitez, and M. Nowakowski for the encouragement and critical suggestions and also thank M. C. Tijero for reading the manuscript. This work was supported by the Coordenação de Aperfeiçoamento de Pessoal de Nível Superior (CAPES).
[1] S. Adler, Phys. Rev. 177, 2426 (1969); J. S. Bell and R. Jackiw, Nuovo Cimento A 60, 49 (1969).

[2] R. Foot, G. C. Joshi, H. Lee, and R. R. Volkas, Mod. Phys. Lett. A 5, 2721 (1990); R. Foot, H. Lee, and R. R. Volkas, J. Phys. G 19, 269 (1993).

[3] K. S. Babu and R. N. Mohapatra, Phys. Rev. D 41, 271 (1990).
[4] J. Sladkowski and M. Zralek, Phys. Rev. D 45, 1701 (1992).

[5] K. S. Babu and R. N. Mohapatra, Phys. Rev. Lett. 63, 938 (1989).

[6] C. Q. Geng and R. E. Marshak, Phys. Rev. D 39, 693 (1989); C. G. Geng, ibid. 41, 1292 (1990); E. Golowich and P. B. Pal, ibid. 41, 3537 (1990); S. Rudaz, ibid. 41, 2619 (1990); M. 
Nowakowski and A. Pilaftsis, ibid. 48, 259 (1993); N. G. Deshpande, University of Oregon Report No. OITS-107, 1979 (unpublished).

[7] K. S. Babu and R. N. Mohapatra, Phys. Rev. D 42, 3866 (1990)

[8] C. A. de S. Pires and O. P. Ravinez, Phys. Rev. D 58, 035008 (1998).

[9] F. Pisano and V. Pleitez, Phys. Rev. D 46, 410 (1992); P. H. Frampton, Phys. Rev. Lett. 69, 2889 (1992).

[10] J. C. Montero, F. Pisano, and V. Pleitez, Phys. Rev. D 47, 2918 (1993); H. N. Long, ibid. 53, 437 (1996).

[11] V. Pleitez, Phys. Rev. D 53, 514 (1996).
[12] F. Pisano, Mod. Phys. Lett. A 11, 2639 (1996).

[13] J. T. Liu and D. Ng, Phys. Rev. D 50, 548 (1994).

[14] J. C. Montero, C. A. de S Pires, and V. Pleitez, Phys. Rev. D (to be published), hep-ph/9812306; F. Pisano, J. A. SilvaSobrinho, and M. D. Tonasse, Phys. Rev. D 58, 057703 (1998); Prashanta Das and Pankaj Jain, ibid. 59, 055011 (1999); P. H. Frampton and Xiao-hu Guan, Mod. Phys. Lett. A 13, 2621 (1998); J. C. Montero, F. V. Pleitez, and M. C. Rodrigues, Phys. Rev. D 58, 097505 (1998); 58, 094026 (1998).

[15] R. Foot, O. F. Hernández, F. Pisano, and V. Pleitez, Phys. Rev. D 47, 4158 (1993).

[16] A. Doff and F. Pisano, hep-ph/9812303. 